\title{
Brain structure and joint hypermobility: relevance to the expression of psychiatric symptoms
}

\author{
J. A. Eccles, F. D. C. Beacher, M. A. Gray, C. L. Jones, L. Minati, N. A. Harrison and H. D. Critchley
}

\begin{abstract}
Summary
Joint hypermobility is overrepresented among people with anxiety and can be associated with abnormal autonomic reactivity. We tested for associations between regional cerebral grey matter and hypermobility in 72 healthy volunteers using voxel-based morphometry of structural brain scans. Strikingly, bilateral amygdala volume distinguished those with from those without hypermobility The hypermobility group scored higher for interoceptive sensitivity yet were not significantly more anxious. Our
\end{abstract}

findings specifically link hypermobility to the structural integrity of a brain centre implicated in normal and abnormal emotions and physiological responses. Our observations endorse hypermobility as a multisystem phenotype and suggest potential mechanisms mediating clinical vulnerability to neuropsychiatric symptoms.

\section{Declaration of interest}

None.
Joint hypermobility is a common but often poorly recognised connective tissue condition. ${ }^{1}$ Individuals with hypermobility are (up to 16 times) overrepresented among those with panic or anxiety disorders. ${ }^{2}$ Hypermobility is also linked to stresssensitive psychosomatic disorders including irritable bowel syndrome, fibromyalgia and chronic fatigue and is associated with hypersensitivity to nociceptive stimuli. ${ }^{1}$ Additionally, individuals with hypermobility often exhibit autonomic abnormalities, typically postural tachycardia syndrome, where there is enhanced cardiovascular reactivity and a phenomenological overlap with anxiety disorders. ${ }^{3}$ Thus, direct and indirect evidence links hypermobility to anxiety and stress-sensitive medical disorders. Within a programme of research motivated to detail the theoretical contribution of central autonomic control to emotion regulation and psychiatric disorders, ${ }^{4,5}$ we performed a voxelbased morphometry (VBM) study of brain magnetic resonance imaging (MRI) scans in participants with and without hypermobility, none of whom had an anxiety disorder.

\section{Method}

High-resolution $(0.9 \mathrm{~mm}$ isometric voxels) structural brain MRI scans (192 sagittal slices, repetition time $11.4 \mathrm{~ms}$, echo time $4.4 \mathrm{~ms}$, inversion time $300 \mathrm{~ms}$ ) were acquired using a Siemens Avanto $1.5 \mathrm{~T}$ scanner (Siemens, Erlangen, Germany) from 72 volunteers, free from clinical anxiety. The participants' characteristics are shown in online Table DS1. Objective measures of hypermobility, anxiety and interoceptive sensitivity (subjective awareness of internal bodily physiological responses $)^{4}$ were acquired using the Beighton scale, Beck Anxiety Inventory (BAI) and Porges Body Perception Questionnaire (PBPQ) respectively. ${ }^{6-8}$ Regional brain volumes were quantified using VBM incorporating diffeomorphic registration (DARTEL) within SPM8 (http://www.fil.ion.ucl.ac.uk/spm/). ${ }^{9}$ Voxel-wise comparisons of spatially normalised grey-matter volume maps of the hypermobile and non-hypermobile groups were performed within a general linear model treating age, gender, handedness and whole brain volume as potentially confounding covariates. Main effects of group, and within-group correlations with hypermobility score, are reported for all brain clusters of $\geqslant 10$ contiguous voxels with significance threshold $P<0.001$ uncorrected. Left and right amygdala were defined a priori regions of interest, using $8 \mathrm{~mm}$ spheres centred on coordinates $(27,-1$, $-19)$ and $(-24,-2,-19)$, based on reported associations between amygdala and anxiety (see e.g. Hayano et al). ${ }^{10}$ We report these results using a stringent family-wise error (FWE) volumecorrected threshold of $P<0.05$. Resting supine heart rate was recorded for $5 \mathrm{~min}$ prior to scanning using a pulse oximeter (Nonin 8600F0; Nonin Medical, Plymouth, Minnesota, USA) and heart rate variability was analysed using the Kubios HRV tool (University of Eastern Finland, Kuopio, Finland).

\section{Results}

Thirty-six participants scored 1 or more on the Beighton scale (the hypermobile group). There was no significant group difference in age, gender or handedness. Bilateral amygdala volume was significantly greater in the hypermobile group than in the non-hypermobile group, meeting both uncorrected $(P<0.001)$ and small-volume corrected FWE thresholds (right amygdala $P=0.014$; left amygdala $P=0.021$ ) (Fig. 1, online Table DS2). Cohen's $d$ effect sizes were large for the amygdala bilaterally (left 0.80 , right 0.85 ). Other group volumetric differences are reported in online Table DS2 and included regions within anterior cingulate and parietal cortices.

Within the hypermobile group, degree of hypermobility correlated positively with the volume of left lateral occipital cortex and negatively with right superior temporal cortex and bilateral inferior parietal cortices (online Fig. DS1, Table DS2). Interestingly, this group scored significantly higher for interoceptive sensitivity (mean PBPQ scores: non-hypermobile 88.4, s.d. $=31.0$; hypermobile 104.3 , s.d. $\left.=32.2 ; t_{(1,70)}=2.13, P=0.018\right)$ and showed a trend towards higher anxiety (non-hypermobile 6.47, s.d. $=7.0$; hypermobile 9.31, s.d. $=9.7 ; t_{(1,70)}=1.42, P=0.08$ ). The relationship between hypermobility and interoceptive sensitivity persisted after controlling for anxiety differences $(P=0.041)$. There was no significant group difference in resting heart rate, standard deviation of interbeat interval, or high (parasympathetic) or low (predominantly sympathetic) frequency components of heart rate variability.

\section{Discussion}

We observed structural differences in key emotion-processing brain regions. Notably, the effect size of this amygdala volume difference was comparable to, or greater than, observations from volumetric studies of clinical psychiatric populations. The hypermobile group also displayed decreased volume within other regions implicated in emotional arousal and attention (anterior cingulate, parietal lobe). Moreover, the degree of hypermobility 
(a)
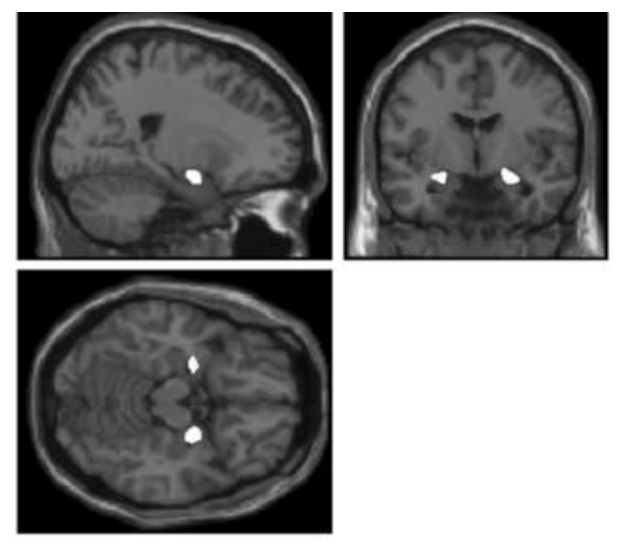

(b)

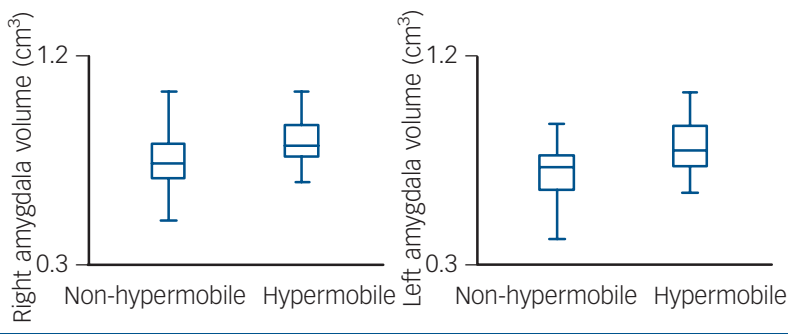

Fig. 1 (a) Regions of grey-matter volume difference in hypermobile participants compared with the non-hypermobile group (white areas; threshold $P<0.001$ uncorrected). (b) Significant group differences in right and left amygdala volumes.

correlated negatively with superior temporal volume, a region implicated in processing social and emotional signals. ${ }^{11}$ Differences in brain structure were not due to overt psychopathology (the hypermobile group only trended toward higher anxiety scores) or basal physiology (no difference was observed in cardiovascular measures at rest).

Our data implicate the amygdala as a likely neural substrate mediating previously reported clinical associations between hypermobility, anxiety and psychosomatic conditions. Speculatively, potential mechanisms include heightened susceptibility of individuals with hypermobility to (threat of) pain and/or a perturbation of autonomic control. Differences in amygdala activity occur in pain disorders including fibromyalgia, irritable bowel syndrome and chronic regional pain syndrome. ${ }^{12}$ Anxiety itself is also linked theoretically to the abnormal generation and mapping of bodily arousal through the engagement of amygdala and insula. ${ }^{5}$ It is also noteworthy that the hypermobility group showed structural differences within anterior cingulate cortex, a central driver of autonomic arousal and a region implicated in the cognitive control of pain and negative emotions. ${ }^{4,12}$ Enhanced interoceptive sensitivity also points to a more finely tuned sensory representation of internal bodily signals within the hypermobile group. Heightened interoceptive awareness is coupled to exaggerated cardiovascular arousal responses. ${ }^{13}$ Moreover, in postural tachycardia syndrome, which commonly occurs with hypermobility (and may have a common basis in collagen variants), heart rate acceleration compensates for dysfunctional vasoconstriction giving rise to physiological symptoms (e.g. palpitations and light-headedness) that are shared with panic and anxiety states. ${ }^{3}$ Such deregulated responses are likely to affect neural processes supporting emotional feelings., ${ }^{4,5}$

Differences in the structural integrity of temporal and parietal cortices may underlie wider behavioural phenotypical expression of hypermobility: abnormalities in superior temporal cortex are also seen in autism. ${ }^{11}$ Inferior parietal cortex can affect proprioceptive awareness and hypermobility is itself linked to dyspraxia. ${ }^{1}$ Our findings suggest that processes compromising function in neurodevelopmental conditions may occur in individuals with hypermobility, putatively enhancing vulnerability to stress and anxiety.

Limitations to our study include the use of a non-clinical sample; interestingly, neuroimaging studies of clinical anxiety groups rarely report enlarged amygdala, suggesting that hypermobility is a distinct phenotype or that amygdala enlargement might be protective in our non-clinical group. Additionally, we used an inclusive definition of hypermobility, although the categorisation of this condition remains debatable. ${ }^{14}$ To conclude, we present the first neuroimaging study of hypermobility that also examines autonomic and interoceptive indices. The observed differences in the structural integrity of specific emotional brain regions provide a starting point for future research into constitutional vulnerabilities to common psychiatric symptoms that have the potential to inform more individually tailored therapeutic approaches.

Jessica A. Eccles, MB BCh, MRCPsych, Department of Psychiatry, Brighton and Sussex Medical School, and Sussex Partnership National Health Service (NHS) Foundation Trust, Sussex Education Centre, Hove; Felix D. C. Beacher, PhD, Marcus A. Gray, PhD, Catherine L. Jones, PhD, Ludovico Minati, MSC, Department of Psychiatry, Brighton and Sussex Medical School, Brighton; Neil A. Harrison, PhD, MRCPsych, Hugo D. Critchley, DPhil, MRCPsych, Department Harrison, PhD, MRCPsych, Hugo D. Critchley, DPhil, MRCPsych, Department
of Psychiatry, Brighton and Sussex Medical School, Brighton, Sussex Partnership NHS Foundation Trust, Sussex Education Centre, Hove and Sackler Centre for Consciousness Science, University of Sussex, Brighton, UK

Correspondence: Dr Jessica Eccles, Psychiatry, Brighton and Sussex Medical school Brighton, BN1 9RR, UK. Email: J.Eccles@bsms.ac.uk

First received 16 Feb 2011, final revision 14 Nov 2011, accepted 19 Dec 2011

\section{Funding}

This work was funded by a Wellcome Trust programme grant (no. 074333) to H.D.C. J.A.E is supported by the National Institute for Health Research and N.A.H. by the Wellcome Trust.

\section{References}

1 Grahame R. Hypermobility: an important but often neglected area within rheumatology. Nat Clin Pract Rheumatol 2008; 4: 522-4.

2 Garcia Campayo J, Asso E, Alda M, Andres EM, Sobradiel N. Association between joint hypermobility syndrome and panic disorder: a case-control study. Psychosomatics 2010; 51: 55-61.

3 Mathias CJ, Low DA, lodice V, Owens AP, Kirbis M, Grahame R. Postural tachycardia syndrome - current experience and concepts. Nat Rev Neurol 2011; 8: 22-34

4 Critchley HD. Psychophysiology of neural, cognitive and affective integration: fMRI and autonomic indicants. Int J Psychophysiol 2009; 73: 88-94.

5 Paulus MP, Stein MB. An insular view of anxiety. Biol Psychiatry 2006; 60: 383-7.

6 Beighton P, Solomon L, Soskolne CL. Articular mobility in an African population. Ann Rheum Dis 1973; 32: 413-8.

7 Beck AT, Epstein N, Brown G, Steer RA. An inventory for measuring clinical anxiety: psychometric properties. J Consult Clin Psychol 1988: 56: 893-7.

8 Porges S. Body Perception Questionnaire. Laboratory of Developmental Assessment, University of Maryland, 1993.

9 Ashburner J. A fast diffeomorphic image registration algorithm. Neurolmage 2007; 38: 95-113.

10 Hayano F, Nakamura M, Asami T, Uehara K, Yoshida T, Roppongi T, et al. Smaller amygdala is associated with anxiety in patients with panic disorder Psychiatry Clin Neurosci 2009; 63: 266-76.

11 Boddaert N, Chabane N, Gervais H, Good CD, Bourgeois M, Plumet MH, et al. Superior temporal sulcus anatomical abnormalities in childhood autism: a voxel-based morphometry MRI study. Neurolmage 2004; 23: 364-9.

12 Tracey I, Bushnell MC. How neuroimaging studies have challenged us to rethink: is chronic pain a disease? J Pain 2009; 10: 1113-20.

13 Herbert BM, Pollatos O, Flor $\mathrm{H}$, Enck $\mathrm{P}$, Schandry R. Cardiac awareness and autonomic cardiac reactivity during emotional picture viewing and mental stress. Psychophysiology 2010; 47: 342-54.

14 Remvig $L$, Jensen DV, Ward RC. Are diagnostic criteria for general joint hypermobility and benign joint hypermobility syndrome based on reproducible and valid tests? A review of the literature. J Rheumatol 2007; 34: 798-803. 